\title{
Death-defying acts: a meeting review on apoptosis
}

\author{
Eileen White \\ Center for Advanced Biotechnology and Medicine, Department of Biological Sciences, Rutgers University, \\ Piscataway, New Jersey 08854 USA
}

Apoptosis, or programmed cell death, is the process by which a cell will actively commit suicide under tightly controlled circumstances. The apoptotic death process is associated with pronounced morphological changes in the cell as well as intranucleosomal DNA fragmentation. These criteria distinguish apoptosis from necrosis, which is cell death that occurs usually in response to physical injury.

The last several years have produced an explosion of both interest in and information about apoptosis, not unlike that which occurred for the cell cycle in the 1980s. In the past, investigators did not appreciate the significance of cell death, and experiments with cell death as an outcome most often ended up in the trash. There was also a lack of molecular reagents with which to study cell death. Now, however, apoptosis is emerging as a key biological regulatory mechanism, possibly no less significant than regulation of cell growth and differentiation.

Apoptosis is essential in many aspects of normal development and is required for maintaining homeostasis. Failure to regulate apoptosis negatively is associated with degenerative diseases, and failure to regulate apoptosis positively is associated with cancer and autoimmune disease. Thus, too much or to little cell death can have catastrophic consequences. Developing molecular approaches to study apoptosis in diverse systems is now the main objective of many laboratories. Some unifying themes have emerged: (1) Apoptosis may arise from incompatible or conflicting growth signals; (2) multiple pathways exist for induction of apoptosis; and (3) apoptosis can be a multistep process. This review is not meant to be comprehensive, but it is an attempt to highlight the most significant and interrelated developments presented by the participants at The Fifth Annual Pezcoller Symposium. ${ }^{1}$

\section{Systems of cell death}

Because apoptosis is such a ubiquitous process, there are as many ways to study it as there are biological systems.

\footnotetext{
1The Fifth Annual Pezcoller Symposium "Apoptosis," Trento, Italy, June 9-11, 1993.
}

In mammalian cells, gene products that either activate or suppress apoptosis have been identified. Of the activators of apoptosis, the product of the p53 tumor suppressor gene has been shown to be clearly responsible for directing apoptosis in some systems, and these results suggest that apoptosis is a cellular defense against cancer (M. Oren, Weizmann Institute; E. White, Rutgers University). p53, however, does not mediate all forms of apoptosis; thus, there are both p53-dependent and -independent pathways (M. Kastan; J. Isaacs; both Johns Hopkins Oncology Center). Deregulation of c-myc protooncogene expression (J. Cleveland, St. Jude Children's Research Hospital; G. Evan, Imperial Cancer Research Fund) and adenovirus ElA oncogene expression (E. White) are associated with stimulation of both cell proliferation and apoptosis. Activation of apoptosis is thereby associated with the deregulation of cell growth control, or perhaps unbalanced cell growth, that would occur in progressing neoplasia. Tumor suppressor genes like p53 may be responsible for responding to abnormal growth situations by initiating apoptosis.

As in all biological systems, where there are activators there are inhibitors. Growth factors and cytokines clearly play an important regulatory role as survival factors (M. Oren; G. Evan; J. Cleveland). Among the first gene products identified to date that act to inhibit apoptosis are oncogenes, of which the human $b c l-2$ protooncogene is the best known example. Overexpression of $b c l-2$ blocks apoptosis and promotes transformation, indicating that escape from apoptosis can be a step in the transformation process. In the immune system and perhaps during development, $b c l-2$ function may be normally required to regulate apoptosis [J. Ashwell, National Institutes of Health (NIH); D. Green, La Jolla Institute Allergy and Immunology; D. Hockenbery, Fred Hutchinson Cancer Research Center]. Another example is the adenovirus E1B oncogene, which encodes two products that function to suppress apoptosis-one that resembles Bcl-2, and the other that directly inhibits p53. Thus, modulation of apoptosis is not only linked with oncogenesis but also with viral pathogenesis (E. White).

The developmental aspects of programmed cell death, which share a number of features with apoptosis, have been explored genetically in the nematode Caenorhabditis elegans regulation [R. Horvitz, Massachusetts In- 
titute of Technology (MIT)]. Both activation and inhi-

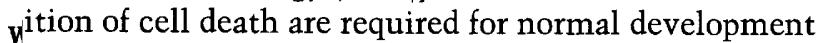
n C. elegans, and the mechanisms may be analogous to hose present in mammalian cells. Apoptosis is also a srominent feature in degenerative diseases of the retina b). Papermaster, University of Texas Health Science iSenter), and in autoimmune diseases (P. Krammer, Gerthan Cancer Research Center; D. Green) The mechanism pf cell death is being studied genetically (J. Cleveland; G. (Ivan; D. Green; D. Hockenbery; R. Horvitz; M. Oren; E. CVhite), biochemically (P. Nicotera, Karolinska Institute; n. Eastman, Dartmouth-Hitchcock Medical Center), and $\mathrm{O}$ relation to cell cycle control /G. Evan; M. Oren; S. Eherwood, Stanford University).

$\mathrm{V}$

$A$

\section{identification of genes that control cell death}

S)ne of the most significant developments in the last everal years has been the identification of gene products I hat regulate cell death by genetic analysis of prorammed cell death in C. elegans. In C. elegans, the linCage and fate of all 1090 cells in the worm are known; s.31 of these cells are destined to die. Thus, programmed tlell death can be evaluated during the normal course of gevelopment and differentiation in C. elegans, and the eegulatory genes can be identified from the analysis of lnutant worms in which cells fail to die or die inapprocriately. The Horvitz group has divided programmed cell death into three stages as defined by mutations (Fig. 1): rl) execution, where cell death occurs through ced-3 and ned-4 function, and is inhibited by ced-9; (2) engulfment, phere the dead cell undergoes phagocytosis by a neighdoring cell, which requires two sets of genes, ced-1, ced(i, ced-7, ced-8, and ced-2, ced-5, ced-10; and, finally, (3) $c$ egradation, where the engulfed cell is degraded, requirung the function of the nuc-1 gene.

b

6

ded-3, ced-4

i] oss-of-function mutations in either ced-3 or ced-4 preent death of all 131 cells during worm development, ctablishing that ced-3 and ced-4 are required for inducng cell death (Ellis and Horvitz 1986). Genetic mosaic Ixperiments have suggested that both gene products unction directly within the dying cell. Surprisingly, the eundead cells" in worms defective for ced-3 or ced-4 iunction go on to differentiate and apparently can funceion, indicating that perturbation of cell death does not flecessarily affect differentiation. Both ced-3 and ced-4 "ave been cloned. ced-4 is expressed in embryonic de$f_{1}$

t:

n

h

igure 1. Regulation of programmed cell eath in C. elegans. velopment, when most cell death occurs, and encodes a novel sequence; part of the predicted Ced-4 63-kD amino acid sequence resembles an EF hand domain, suggesting that it may be a calcium-binding protein. However, there are no biochemical data yet that show whether Ced-4 binds calcium or whether this motif is related to Ced-4 function. ced-3 is also expressed during embryogenesis and encodes a predicted central serine-rich region that acts in a dominant-negative fashion to block cell death when expressed as a gene fusion with lacZ. Many of the mutations affecting ced-3 function are found in the carboxyl terminus, suggesting that this region of the protein is important for function.

\section{ced-9}

The ced-9 gene product has been identified as a negative regulator of ced-3 and ced-4, by blocking cell death (Hengartner et al. 1992). ced-9 gain-of-function mutations prevent all cell deaths that occur during normal worm development, similar to the phenotype of ced-3 and ced-4 loss-of-function mutations. ced-9 loss-of-function mutations cause death of the entire organism, indicating that ced-9 is required actively and continually to prevent all cell deaths within the animal. Other modifiers of ced-3 and ced-4 function have also been identified and are designated ces- 1 and ces- 2 for cell death specification. ces-2 and ces-1 act to prevent death of a subset of cells in the worm, imparting cell type specificity to cell death, a theme reiterated in studies in mammalian cells (see below).

ced-1, ced- 6 , ced- 7 , ced-8, ced- 2 , ced- 5 , ced- 10

Once cell death has taken place, the organism must dispose of the dead cell, or corpse, a process probably very important in higher organisms for preventing an inflammatory reaction. The first step in the disposal process is phagocytosis of the corpse by another cell, and this requires the function of the engulfment genes. Engulfment occurs independently and downstream of execution, because corpses remain in the worm if engulfment does not take place. The engulfment genes have been classified into two sets. Mutation of any one gene alone does not impair engulfment dramatically, but mutations in one of each of the two sets does. This functional redundancy suggests the existence of two parallel pathways.

nuc-1

Once engulfed, the corpse is degraded. This final step in 
the process requires the function of the nuc-1 gene. Like the engulfment genes, nuc-1 function is not required for cell death and is a distinct downstream event. The nuc-1 gene product controls the activity of a nuclease required to degrade the DNA in the engulfed corpse.

These studies in C. elegans demonstrate that positive and negative regulation of cell death is an essential aspect of normal development, that it is a multistep process, and that the mechanism is likely to be functionally conserved in higher organisms. Presumably, functional equivalents, if not homologs to the ced genes exist in mammalian cells. To date, the identification of genes that regulate apoptosis in mammalian cells has derived from several sources: oncogenes, tumor suppressor genes, and viruses.

\section{p53}

M. Oren originally observed that ectopic expression of wild-type p53 in the myeloid leukemic cell line M1, which is devoid of endogenous p53, induced apoptosis (Yonish-Rouach et al. 1991). These observations raised the provocative possibility that p53 may function as the ultimate tumor suppressor by producing cell death. This experiment utilized the murine temperature-sensitive, dominant-negative p53(val135) mutant, which has properties of mutant $\mathrm{p} 53$ at $37.5^{\circ} \mathrm{C}$ and those of the wild-type form at $32^{\circ} \mathrm{C}$. M1 cells expressing the $\mathrm{p} 53$ (val135) protein grow rapidly at $37.5^{\circ} \mathrm{C}$ when p53 is mutant and undergo apoptosis at $32^{\circ} \mathrm{C}$ when p53 is wild type. There was no evidence for cell cycle arrest at $32^{\circ} \mathrm{C}$ before apoptosis, indicating that a cell cycle block was not a prerequisite to apoptosis by p53. However, there may be a decision point to initiate apoptosis in late $G_{1}$ because cells in this phase of the cell cycle are the first to undergo apoptosis.

The induction of p53-dependent apoptosis in M1 cells, which are highly responsive to interleukin-6 (IL-6), was specifically suppressed by IL- 6 . In contrast to the continued cell cycle progression and apoptosis at $32^{\circ} \mathrm{C}$, without IL-6, wild-type $\mathrm{p} 53$ produced $\mathrm{G}_{1}$ arrest in the presence of IL-6. One interpretation of these results is that a way to escape apoptosis is to exit from the cell cycle, a situation promoted by IL-6 (Fig. 2). Alternatively, IL-6 and perhaps other survival factors may alter the activity of p53 ranging from induction of apoptosis to induction of growth arrest. Nonetheless, wild-type p53 renders Ml cells fac-

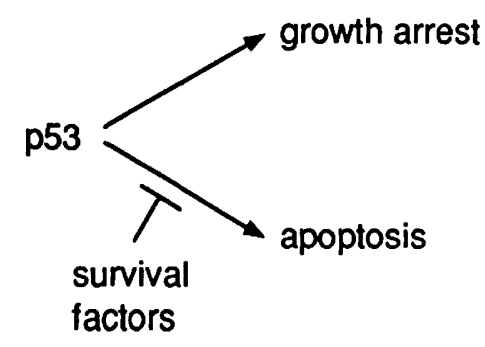

Figure 2. Regulation of apoptosis by p53. tor dependent, which is an effective way to control inappropriate cell proliferation.

The ectopic expression of large amounts of wild-type p53 is an artificial situation for induction of apoptosis, thus raising the question of whether endogenous p53 functions similarly. To address this issue, the IL-3-dependent myeloid cell line 32D, which expresses wildtype p53, was examined (M. Oren). IL-3 withdrawal normally induces apoptosis in 32D cells. Depletion of endogenous wild-type p53 protein levels using antisense constructs, or blocking p 53 function with the dominantnegative mutant p53(val135) at $37.5^{\circ} \mathrm{C}$, delayed apoptosis substantially upon IL-3 withdrawal. Furthermore, vast overexpression of wild-type p53 did not induce apoptosis unless IL-3 was withdrawn. Thus, endogenous wild-type p53 function is required for the induction of apoptosis upon survival factor withdrawal, but different factors act to promote survival in different cell types.

To rule out a contribution of the gain-of-function of mutant p53 to the regulation of apoptosis, $M$. Oren utilized a carboxy-terminal segment of p53 that encompasses the oligmerization domain and will competitively inhibit p53 while lacking the gain-of-function activity. This dominant-negative form of p53 was capable of preventing apoptosis by factor withdrawal, a result supported by observations with fibroblasts nullizygous for p53 (see below).

The mechanism by which survival factors such as IL-3 and IL- 6 prevent p53-dependent apoptosis is only beginning to be addressed (M. Oren). IL- 6 treatment of M1 cells does not produce conformational changes in p53, nor is the localization affected. IL- 6 does not change the levels of $b c l-2$ expression, another possible means of overcoming apoptosis. p53 not only can regulate apoptosis but cell cycle progression and transcription as well. The relationship of these activities to the control of p53dependent apoptosis by survival factors will be important to determine.

c-myc

c-myc is an early response gene that is often associated with normal cell proliferation but is oncogenic when its expression is deregulated. Several laboratories (J. Cleveland; G. Evan; D. Green) have been examining induction of apoptosis by deregulation of c-myc expression in mammalian cell lines derived from hematopoietic cells or fibroblasts. Normally, c-myc expression is induced in proliferating cells following mitogenic stimulation and is down-regulated following factor withdrawal, whereupon cells commonly arrest in $\mathrm{G}_{1}$. Constitutive, unregulated c-myc expression, which prevents down-modulation of c-myc levels upon factor withdrawal, produces continued DNA synthesis and apoptosis but not growth arrest. The inability of cells with deregulated c-myc to arrest upon factor deprivation suggests that apoptosis is caused by incompatible growth signals resulting from the signal to grow from myc plus the signal to arrest from factor withdrawal. These conflicting growth situations may be irreconcilable, causing cell death. Pertur- 
bation of the cell cycle in an irreparable way may be one way to produce cell death. S. Sherwood presented evidence that perturbation of the cell cycle with low doses of colchicine, hydroxyurea, or aphidicolin alters the levels of specific cyclins and leads to apoptosis.

The transforming region of c-myc is responsible for induction of apoptosis and provides a direct association among cell proliferation, transformation, and apoptosis, even though apoptosis by c-myc does not appear to occur at a particular phase of the cell cycle. Because c-myc produces both proliferation and apoptosis, myc expression may require tight regulation during normal cell growth. The specific factor required for factor-dependent survival of cells expressing deregulated myc is determined by the cell type (i.e., IL-3 in IL-3-responsive hematopoietic cells, IGF-1 in Rat-1 fibroblasts). This may represent a safety mechanism for checking inappropriate cell proliferation involving $m y c$ and is analogous to the factor-dependent state created by expression of $\mathrm{p} 53$.

Survival signals for myc can come in the form of growth factors or can be encoded by genes with products that function to inhibit apoptosis. $b c l-2$ has been shown to inhibit apoptosis when myc expression is deregulated and will cooperate with myc to transform cells (Vaux et al. 1988; Bissonnette et al. 1992; Fanidi et al. 1992). More recently, v-raf has been found to suppress apoptosis by myc to the same extent as $b c l-2$, producing arrest in $\mathrm{G}_{1}$ (J. Cleveland). Similarly v- $a b l$ will suppress apoptosis by c-myc (D. Green). Perhaps v-raf and v-abl, as kinases, merely mimic survival signals that cells would normally receive at the cell surface to suppress apoptosis (Fig. 3).

\section{Adenovirus E1A}

ElA oncogene expression induces both proliferation and apoptosis and is consequently insufficient for transformation of primary rodent cells (E. White). Analysis of E1A mutants has shown cosegregation of induction of proliferation, transformation, and apoptosis, providing a link between the deregulation of cell growth control by ElA and apoptosis, analogous to the observations with deregulated c-myc.

Induction of apoptosis by E1A is clearly p 53 dependent. E1A expression produces p53 accumulation in cells that subsequently undergo apoptosis. Rare transformants arising from transfection of primary cells with E1A alone have been selected for resistance to apoptosis

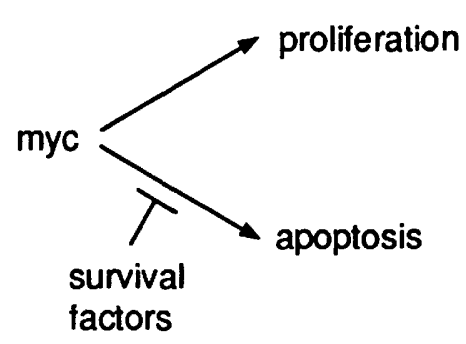

Figure 3. Regulation of apoptosis by c-myc. and have also acquired p53 mutations with high frequency. Dominant-interfering mutant forms of p53 will suppress apoptosis by E1A and permit transformation of primary rodent cells. Cells transformed with E1A plus the temperature-sensitive p53(val135) mutant undergo apoptosis when p53 assumes the wild-type conformation. Thus, wild-type p53 function is required for induction of apoptosis by E1A. Much like the situation created by high levels of myc in the absence of growth factors, E1A expression strongly stimulates cell cycle progression in the presence of high levels of p53 that are cytostatic. The delivery of conflicting growth signals by E1A and p53 is currently the most likely cause of apoptosis (Debbas and White 1993).

\section{bcl-2}

$b c l-2$ overexpression was initially reported to promote survival of promyeloid cells upon IL-3 withdrawal, which causes apoptosis (Vaux et al. 1988; Hockenbery et al. 1990). bcl-2 has since been demonstrated to block apoptosis in many assays and can promote oncogenesis in animal models. The focus now is on the mechanism and identification of the pathways in which $b c l-2$ normally functions in development. The mechanism of $b c l-2$ action has not yet been elucidated. The protein has been reported by different groups to reside either in $\mathrm{mi}$ tochondrial or nuclear envelope and endoplasmic reticulum membranes. These discrepancies may be the consequence of cell type variations or the use of anti-Bcl-2 antibodies that recognize different epitopes. D. Hockenbery pointed out that deletion of the last 20 amino acids of Bcl-2, which contains the putative membrane-spanning region, caused Bcl-2 to be entirely cytoplasmic while it retained substantial functional activity. The specific intracellular location of $\mathrm{Bcl}-2$, therefore, may not be critically important for Bcl-2 function.

The pathways in which $b c l-2$ functions to inhibit apoptosis are being investigated with the use of transgenic mice in which Bcl-2 overexpression is directed at different tissues. Targeted expression of Bcl-2 to B cells has profound effects on extending B-cell memory and produces lymphomas. In T cells, the role of Bcl-2 in apoptosis is less clear. Thymocytes from transgenic mice that overexpress Bcl-2 in the thymus are resistant to the induction of apoptosis by glucocorticoids, anti-CD-3 antibodies, and $\gamma$-irradiation, but the capacity to undergo negative selection is variable (Sentman et al. 1991; Strasser et al. 1991). This may be attributable to the existence of multiple pathways for induction of apoptosis, only some of which involve $b c l-2$, variations in genetic background, or the level of Bcl-2 expression.

Bcl-2 function has begun to be explored beyond the immune system. As a first step, D. Hockenbery reported on the distribution of Bcl-2 protein in human tissue. $b c l-2$ mRNA is found in postmitotic neurons, brain, retina, epithelium, intradigital tissue of limb buds, and in the basal level in skin but not in more differentiated layers. The exclusion of $b c l-2$ expression from more terminally differentiated tissues suggests that apoptosis 
needs to be actively suppressed when growth pathways are activated, such as in proliferating but not terminally differentiated cells. $b c l-2$ expression is also required to suppress apoptosis in cells where growth pathways are activated by myc and E1A, indicating that the same paradigm may exist in oncogenesis.

Another approach to $b c l-2$ function is to ectopically express $b c l-2$ in other tissues. Bcl-2 expression has been directed to the mouse mammary gland using the mouse mammary tumor virus (MMTV) promoter in transgenic animals to examine the ability of $b c l-2$ to influence hormone-dependent tissue induction and regression (D. Hockenbery). MMTV-bcl-2 transgenic mice display less tissue regression postlactation than do control animals. In contrast to mice that have targeted overexpression of $b c 1-2$ in hematopoietic cells, the MMTV-Bcl-2 mice do not develop breast tumors.

\section{Adenovirus $E 1 B 19 \mathrm{~K}$ and $55 \mathrm{~K}$ proteins}

The ElB oncogene products inhibit apoptosis induced by ElA expression (E. White). This function is required to prevent the premature death of the host cell and impairment of virus yield in productively infected human cells. Coexpression of an inhibitor of apoptosis such as E1B or $b c l-2$ with ElA is also necessary for transformation of primary rodent cells to take place. The E1B gene encodes two distinct polypeptides (19K and $55 \mathrm{~K}$ ), both of which independently suppress apoptosis by E1A and cooperate with E1A in transformation assays. In adenovirus, induction of cell proliferation must be coupled to suppression of apoptosis for transformation. The parallels between E1A and c-myc and between $b c l-2$ and E1B are striking and suggest that common mechanisms may be associated with the induction and suppression of apoptosis during oncogenesis.

Expression of the E1B $19 \mathrm{~K}$ or $55 \mathrm{~K}$ proteins will inhibit E1A-induced p53-dependent apoptosis by direct and indirect mechanisms. The $55 \mathrm{~K}$ protein complexes with p53 and directly inhibits p53 function (Yew and Berk 1992). The E1B 19K protein will inhibit apoptosis completely when expressed in cell lines derived from primary rodent cells transformed by E1A plus the temperature-sensitive p53(val135) mutant, when p53 assumes the wild-type conformation (Debbas and White 1993). In cell lines where p53-dependent apoptosis is blocked by the E1B 19K protein, cell death is averted and growth is arrested. Therefore, the $19 \mathrm{~K}$ protein promotes the growth arrest rather than apoptotic activity of p53, as does IL- 6 in M1 cells. This indicates that the growth arrest and apoptotic functions of p53 are distinct and separable. The $19 \mathrm{~K}$ protein does not detectably bind $\mathrm{p} 53$, nor are the levels or localization of p53 affected. The E1B $19 \mathrm{~K}$ protein will, however, modify the transcriptional activity of p53 detected in CAT assays, suggesting a relationship between the regulation of gene expression and apoptosis.

The functional interchangeability of the E1B $19 \mathrm{~K}$ and $b c l-2$ genes in transformation assays with E1A and the dependence of E1A-associated apoptosis on p53 have in- dicated that Bcl-2 may block p53-dependent apoptosis. Bcl-2 expression in E1A plus p53(val135)-transformed cells will prevent the induction of apoptosis when p53 assumes the wild-type conformation, as does the E1B $19 \mathrm{~K}$ protein. The oncogenic and anti-apoptotic activity of Bcl-2 and the E1B $19 \mathrm{~K}$ protein may ultimately be attributed all or in part to bypassing the function of $\mathrm{p} 53$ (Fig. 4).

The E1B $19 \mathrm{~K}$ protein may be the viral equivalent to Bcl-2. Both are membrane proteins that block induction of apoptosis by tumor necrosis factor (TNF) $\alpha$ and Fas antigen, and $b c l-2$ expression will compensate for the absence of E1B $19 \mathrm{~K}$ gene function during infection and transformation. So far, the E1B $19 \mathrm{~K}$ and Bcl-2 proteins are functionally qualitatively indistinguishable. There are also limited similarities in the amino acid sequences of the two proteins that suggest a related biochemical function.

\section{Pathways that regulate cell death}

There is substantial evidence to suggest that multiple pathways exist for induction of apoptosis with numerous proximal and distal points at which inhibitors could intervene. Either multiple pathways for induction of apoptosis could exist within the same cell or only certain pathways may be activated in specific cell types. p53 has been shown clearly to cause growth arrest or apoptosis in different situations that may depend on the source of cells (primary cells or cell lines), the cell type, or both. Many stable cell lines may have been actually selected to have shut down apoptosis just by virtue of being cell lines. This could be the result of loss of wild-type p53 or other regulators of apoptosis not yet determined. $M$. Kastan has addressed the issue of the growth arrest versus apoptosis functions of $\mathrm{p} 53$ by examining response of p53 in different cell types after DNA damage induced by $\gamma$-irradiation. In cell lines p53 can act as a cell cycle control check point in response to DNA damage by accumulating in response to DNA damage and producing cell cycle arrest at $G_{1}$ (Kastan et al. 1992). Cell lines lacking wild-type p53 function bypass the $G_{1}$ checkpoint, progress through $S$ phase, and accumulate in $G_{2}$. To rule out any complications or unknown contributory factors that may preexist in stable cell lines, mouse embryo fibroblasts from homozygous wild-type, heterozy-

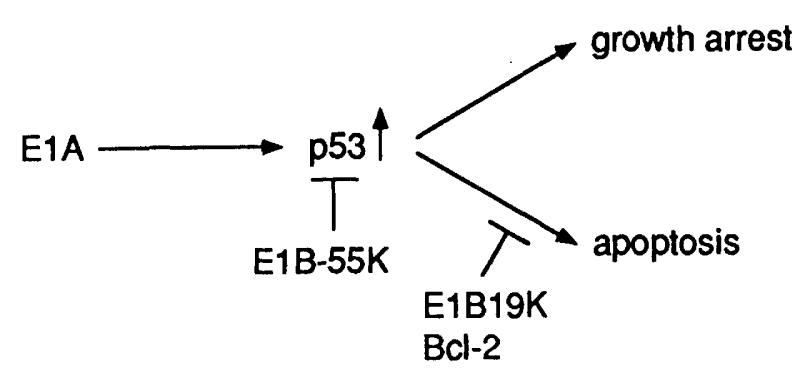

Figure 4. Regulation of p53-dependent apoptosis by E1A, E1B, and Bcl-2. 
gous, and nullizygous p53 mice were examined. Fibroblasts derived from the homozygous and heterozygous wild-type p53 mice responded to DNA damage with growth arrest as did cell lines that contained wild-type p53. The fibroblasts from the nullizygous p53 animals, however, did not display $G_{1}$ arrest. Thus, in normal fibroblasts, p53 functions to produce growth arrest in response to DNA damage. Interestingly, thymocytes from these same homozygous and heterozygous wild-type p 53 animals undergo apoptosis in response to $\gamma$-irradiation, whereas thymocytes from the p53 nullizygous animals do not (Clarke et al. 1993; Lowe et al. 1993). Therefore, different cell types respond differently to DNA damage and p53 accumulation with either apoptosis /thymocytes) or growth arrest (fibroblasts) (Fig. 5). In both cell types $\gamma$-irradiation, produces p53 accumulation to the same extent so that cell-type-specific differences cannot be immediately explained on the basis of differential p 53 levels.

There is also mounting evidence for the existence of both p53-dependent and -independent apoptotic pathways. Mice nullizygous for p53 develop normally but have a higher incidence of cancer (Donehower et al. 1992). p53 is not likely to be required for apoptotic processes in development but may be extremely important as a defense mechanism by acting to induce apoptosis in emerging transformed and perhaps virus-infected cells. p53 is required for thymocytes to undergo apoptosis in response to DNA damage but is not required for glucocorticoid-induced apoptosis (Clarke et al. 1993). p53 is also dispensable for apoptosis in response to serum but not IL-6 deprivation in M1 cells, indicating that p53dependent and -independent pathways can exist in the same cell (M. Oren). Bcl-2 and E1B 19K proteins can block apoptosis that is both p53 dependent and independent, suggesting that both function downstream of $\mathrm{p} 53$ and inhibit multiple pathways (E. White).

$\mathrm{J}$. Isaacs addressed the involvement of p53 in an apoptotic pathway in regressing prostate. Prostatic glandular cells are exquisitely androgen dependent for survival. Androgen ablation (castration) produces massive cell death of glandular cells by apoptosis. Glandular cells are predominantly not cycling and undergo apoptosis in the presence of cell cycle inhibitors, suggesting that cell cycle progression is not required for apoptosis. Androgen ablation in p53 nullizygous mice still produces prostate regression by apoptosis, demonstrating another example of a p53-independent pathway (Berges et al. 1993).
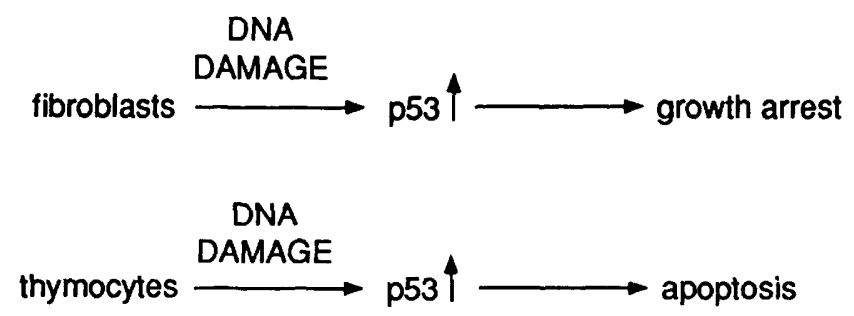

Figure 5. Cell type specificity of p53 function.
Pathways for regulation of apoptosis in the immune system, in which apoptosis is part of the normal differentiation, are in the process of being defined (J. Ashwell). Random rearrangement of T-cell receptor (TCR) ligandbinding-chain gene segments generates diversity of the immune cell repertoire but also receptors that recognize self-antigens. Autoreactive $\mathrm{T}$ cells with high-avidity anti-self in the context of major histocompatability complex $(\mathrm{MHC})$ molecules are eliminated by negative selection by a process that resembles apoptosis. The population of thymocytes with TCRs with moderate avidity for self-antigen/MHC recognition are protected from death and are destined to undergo positive selection and mature into effector $\mathrm{T}$ cells. Thus, strong TCR-ligand interactions may be the signal for the cells to die. Thymocytes with little to no avidity for self, or those with aberrantly rearranged TCRs, are also eliminated by apoptosis in a process that may involve glucocorticoids. J. Ashwell suggested that positive selection may be the combinatorial effect of dual signals from the TCR and glucocorticoid receptor, on the basis of the observation that in T-cell hybridomas and thymic organ cultures, glucocorticoid treatment and TCR occupancy are mutually antagonistic. Signaling through the steroid receptor (glucocorticoids) causes $\mathrm{T}$ cells with low avidity for antigen to die, whereas signaling through the TCR causes T cells with high antigen avidity to die. Signals received from both the TCR and the steroid receptor are mutually antagonistic, causing cells with moderate antigen avidity to live and to be positively selected. Thus, two independent receptor signals are required to escape cell death. Identification of the regulators of apoptosis downstream of the receptors should be informative. Glucocorticoid-mediated apoptosis is clearly independent of $\mathrm{p} 53$ and occurs in thymocytes nullizygous for p53 (Clarke et al. 1993). However, engagement of the TCR in activation-induced apoptosis in T-cell hybridomas does require $m y c$ and is blocked by $m y c$ antisense oligonucleotides that down-regulate Myc protein levels (Shi et al. 1992).

Another important component in regulation of apoptosis in the immune system is the cell-surface antigen Fas, which is a member of the TNF and nerve growth factor receptor superfamily (P. Krammer). Fas antigen is expressed constitutively in a variety of epithelial cells and subpopulations of peripheral $\mathrm{B}$ and $\mathrm{T}$ cells. The cross-linking of Fas antigen on the cell surface with antiFas antibodies such as anti-APO-1 causes apoptosis. A direct connection between Fas antigen function and regulation of immune function was provided by lpr mice, which have mutations in Fas antigen and display a lymphoproliferative disorder with double-negative $T$ cells, and autoantibody production, which resembles the human disease, systemic lupus erythematosis (WatanabeFukunaga et al. 1992). The lack of Fas antigen expression in $1 p r$ mice might impair apoptosis during negative selection and peripheral selection in $\mathrm{T}$ cells as well as B-cell apoptosis. P. Krammer and D. Green presented evidence that negative selection appears normal in $1 p r$ mice but peripheral selection is impaired. 


\section{Mechanisms of cell death}

Whether apoptosis is caused by incompatible growth signals, the absence of survival signals, unbalanced growth, or insufficient growth stimulatory signals, the mechanism by which the cell executes itself is the next step in the process. The C. elegans system has illustrated that death is a multistep process that is tightly regulated by both ubiquitous and cell-type-specific gene products. At this time, there is no reason to think that regulation of apoptosis in mammalian cells will otherwise differ. myc and E1A functionally resemble ced-3 and ced-4, whereas Bcl2 and E1B 19K functionally resemble ced-9. With the genes in hand, the biochemical mechanism of induction and suppression of apoptosis is an attainable goal. Yet there is still experimentation and speculation as to the means by which the cell commits suicide, in part because the biochemical aspects of cell death are fraught with the problem of cause versus effect in dying cells.

Events common to cells undergoing apoptosis are the profound cytoplasmic boiling, the condensation of chromatin, and the endonucleolytic cleavage of DNA. These events are likely to represent discrete steps in the cell destruction process. Although there are increasing numbers of reports that cell death can occur without DNA fragmentation, there is still speculation about the mechanism of DNA destruction. Two theories prevail: Either the nuclease activity is induced, or the DNA substrate becomes available to a preexisting nuclease. P. Nicotera presented evidence that calcium-dependent unfolding of chromatin may be important for apoptosis. In permeabilized thymocytes, calcium produces localized unfolding of chromatin and DNA fragmentation. Polyamines, such as spermine, that suppress chromatin unfolding can inhibit DNA fragmentation. A. Eastman is a proponent of the nuclease induction model and has purified a nuclease (DNase II) from Chinese hamster ovary cells that is activated by ionophore induction of apoptosis. In the cell lines examined, apoptosis correlated with a drop in $\mathrm{pH}$ and not with elevated calcium levels. DNase II is activated by low $\mathrm{pH}$, suggesting that acidosis may produce nuclease activation and DNA cleavage. Positive identification of the nuclease awaits cloning of the nuclease gene. Different nucleases may be involved in different cell types, and both nuclease induction and substrate availability may ultimately be relevant.

\section{Future prospects}

The next year promises some interesting advances in the area of apoptosis. With some of the genes that regulate apoptosis now identified, the biochemical mechanism of apoptosis, identification of the pathways, and establishment of the role of apoptosis in normal development can be addressed. A central experiment establishing the role of apoptosis in normal development would be gene knockout experiments in mice. These have already been done for p53 and some forms of myc, and work is in progress for $b c l-2$. Furthermore, the availability of cells from nullizygous animals will also provide a source of otherwise normal tissue lacking the expression of a particular gene, circumventing the problem of unknown genetic changes that may exist in stable cell lines.

The identification of new gene products that function to regulate apoptosis is likely to be an expanding area. The number of oncogenes and tumor suppressor genes that either induce or suppress apoptosis is likely to increase. Selection strategies for cells resistant to apoptosis will probably turn up new gene products that regulate apoptosis, some perhaps related to $b c l-2$. Reports of viruses and viral genes that regulate apoptosis are also increasing. The characterization of the products of these viral genes will be a fertile area for the identification of control elements in the apoptosis process. The identification of mammalian homologs of the C. elegans regulators of cell death will also be informative.

The mechanism of inhibition of apoptosis by Bcl-2 and the E1B 19K proteins awaits the definition of their biochemical mode of action. This will no doubt be facilitated by the identification of the cellular machinery with which they interact. There is, in addition, the question of how survival factors act. One possibility is that they affect the levels or activity of myc, bcl-2, or p53. Likewise, identification of the activities of myc, ElA, and Fas antigen that produce apoptosis will provide the complete picture of regulation of apoptosis in these settings.

p53 has emerged as a major regulator of apoptosis that is extremely significant in oncogenesis but probably not in development. IL-6, E1B 19K, and Bcl-2 appear to alter the activity of $\mathrm{p} 53$ ranging from induction of apoptosis to growth arrest. Determination of how the activity of p53 is modulated will be important. Alterations in the phosphorylation state, half-life, DNA binding, and transcriptional activity are known to affect p53 function and may be altered during activation or suppression of apoptosis. Finally, multiple pathways for apoptosis exist, and a role for $\mathrm{p} 53$ in a subset of pathways has been established. How pervasive is p53, and is its role restricted to apoptosis in oncogenesis and DNA damage? Is p53 downstream of myc?

Finally, one would like to know how apoptosis can be manipulated to control human disease. The ability to use specific drugs to turn on apoptosis in cancer cells or turn off apoptosis in degenerative diseases will be invaluable. These are some of the short- and long-term goals and expectations, and we look forward to the next chapter in apoptosis research.

\section{References}

Berges, R.S., Y. Furuya, L. Remington, H.F. English, T. Jacks, and J.T. Isaacs. 1993. Cell proliferation, DNA repair, and p53 function are not required for programmed cell death of prostatic glandular cells induced by androgen ablation. Proc. Natl. Acad. Sci. (in press).

Bissonnette, R.P., F. Echeverri, A. Mahboubi, and D. Green. 1992. Apoptotoc cell death induced by $c-m y c$ is inhibited by bcl-2. Nature 359: 552-554.

Clarke, A.R., C.A. Purdie, D.J. Harrison, R.G. Morris, C.C. Bird, M.L. Hooper, and A.H. Wyllie. 1993. Thymocyte apoptosis induced by p53-dependent and independent pathways. $\mathrm{Na}$ - 
White

ture 362: 849-852.

Debbas, M. and E. White. 1993. Wild-type p53 mediates apoptosis by E1A which is inhibited by E1B. Genes \& Dev. 7: 546-554.

Donehower, L.A., M. Harvey, B.L. Slagle, M.J. McArthur, J.C.A. Montgomery, J.S. Butel, and A. Bradley. 1992. Mice deficient for p53 are developmentally normal but suceptible to spontaneous tumors. Nature 356: 215-221.

Ellis, H. and H.R. Horvitz. 1986. Genetic control of programmed cell death in in the nematode C. elgans. Cell 44: 817-829.

Fanidi, A., E.A. Harrington, and G. Evan. 1992. Cooperative interaction between $\mathrm{c}$-myc and $\mathrm{bcl}-2$ proto-oncogenes. $\mathrm{Na}$ ture 359: 554-556.

Hengartner, M.O., R.E. Ellis, and H.R. Hovitz. 1992. Caenorhabditis elegans gene ced-9 protects cells from programmed cell death. Nature 356: 494-499.

Hockenbery, D., G. Nuñez, C. Milliman, R.D. Schreiber, and S. Korsmeyer. 1990. Bcl-2 is an inner mitochondrial membrane protein that blocks programmed cell death. Nature 348: 334-336.

Kastan, M.B., Q. Zhan, W.S. El-Deiry, F. Carrier, T. Jacks, W.V. Walsh, B.S. Plunkett, B. Vogelstein, and A.J. Fornace. 1992. A mammalian cell cycle checkpoint pathway utilizing p53 and GADD45 is defective in ataxia-telangiectasia. Cell 13: 587597.

Lowe, S.W., E.M. Schmitt, S.W. Smith, B.A. Osborne, and T. Jacks. 1993. p53 is required for radiation-induced apoptosis in mouse thymocytes. Nature 362: 847-849.

Sentman, C.L., J.R. Shutter, D. Hockenbery, O. Kanagawa, and S.J. Korsmeyer. 1991. bcl-2 inhibits multiple forms of apoptosis but not negative selection in thymocytes. Cell 67: 879888.

Shi, Y., J.M. Glynn, L. Guilbert, T.G. Cotter, R.P. Bissonnette, and G.D.R.. 1992. Role for $c$-myc in activation-induced apoptotic cell death in $\mathrm{T}$ cell hybridomas. Science 257: 212214.

Strasser, A., A.W. Harris, and S. Cory. 1991. bcl-2 transgene inhibits $\mathrm{T}$ cell death and perturbs thymic self-censorship. Cell 67: 889-899.

Vaux, D.L., S. Cory, and T.M. Adams. 1988. Bcl-2 promotes the survival of haemopoietic cells and cooperates with c-myc to immortalize pre-B cells. Nature 335: 440-442.

Watanabe-Fukunaga, R., C.I. Brannan, N.G. Copeland, N.A. Jenkins, and S. Nagata. 1992. Lymphoproliferation disorder in mice explained by defects in Fas antigen that mediates apoptosis. Nature 356: 314-317.

Yew, P.R. and A.J. Berk. 1992. Inhibition of p53 transactivation required for transformation by adenovirus early $1 \mathrm{~B}$ protein. Nature 357: 82-85.

Yonish-Rouach, E., D. Resnitzky, J. Lotem, L. Sachs, A. Kimchi, and M. Oren. 1991. Wild-type p53 induces apoptosis of myeloid leukaemic cells that is inhibited by interleukin-6. $\mathrm{Na}$ ture 352: 345-347. 


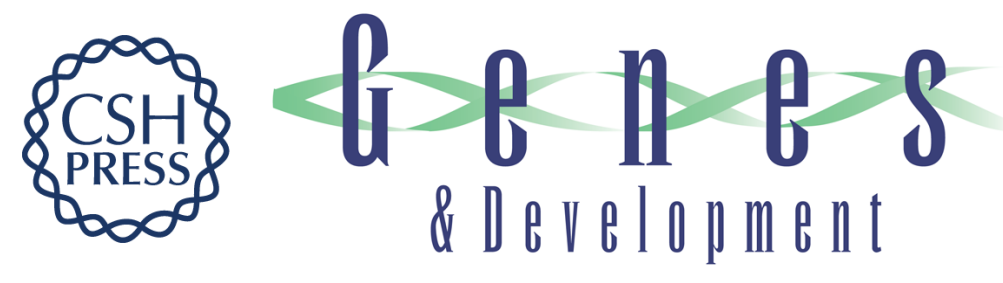

\section{Death-defying acts: a meeting review on apoptosis.}

E White

Genes Dev. 1993, 7:

Access the most recent version at doi:10.1101/gad.7.12a.2277

References This article cites 17 articles, 2 of which can be accessed free at: http://genesdev.cshlp.org/content/7/12a/2277.full.html\#ref-list-1

License

Email Alerting Receive free email alerts when new articles cite this article - sign up in the box at the top Service right corner of the article or click here.

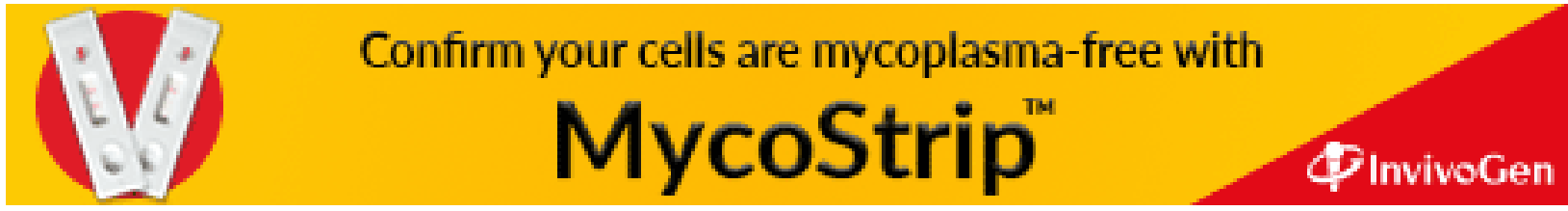

